\title{
Retrograde pyramidal tract degeneration in a patient with cervical haematomyelia
}

\author{
TORU YAMAMOTO, MASAHIRO YAMASAKI, TERUKUNI IMAI \\ From the Department of Neurology, Kitano Hospital and Neurological Centre, Tazuke-Kofukai Medical \\ Research Institute, Osaka, Japan
}

SUMMARY Retrograde pyramidal tract degeneration has been described only very rarely in the human central nervous system. In most of these cases the thoracic or cervical corticospinal tracts were shown to have degenerated following long-standing, lower spinal cord lesions. In a 67 year old man, who lived 2 years following the rupture of a mid-cervical cavernous angioma, we observed such degeneration which reached as high as the pons. This axonal dissolution was much less manifest above the ponto-medullary junction. Large pyramidal cells of Betz were not identified in the precentral gyrus, suggesting that the parental soma of the damaged axons had undergone atrophic changes. Furthermore, the involvement of the so-called aberrant pyramidal tract in the pontine medial lemniscus indicated that retrograde degeneration had occurred there as well.

Retrograde tract degeneration occurring in the proximal portion of an injured nerve is regarded as an exceptional and controversial event, in contrast to the well known axonal reaction observed in neuronal cytoplasm.' Some rare examples of this change in the human corticospinal tract were reported recently by Fishman who examined the chronic changes in posttraumatic paraplegic patients. ${ }^{2}$ According to Bronson et al who reported a similar case in $1978,{ }^{3}$ there had not been any article dealing with this subject in the human being since that of Hunt in $1904 .^{4}$ Partly because of the limited number of reported cases, the histological characteristics of this retrograde change have not yet been detailed.

In a case of cervical haematomyelia who survived for 2 years after the haemorrhage, we now report definite changes in the pyramidal tract as high as the mid-pons. In addition, we found degeneration in the so-called aberrant pyramidal tract ${ }^{-8}$ in the pontine medial lemniscus.

\section{Case report}

A 66 year old man with no contributory past medical history developed weakness of the right arm following 1 week of right shoulder pain. After several hours the weakness spread

Address for reprint requests: Toru Yamamoto, MD, Department of Neurology, Kitano Hospital and Neurological Center, TazukeKofukai Medical Research Institute, 13-3 Kamiyama-cho, Kita-ku, Osaka 530, Japan.

Received 17 June 1988 and in revised form 28 October 1988. Accepted 28 October 1988 to the right lower extremity and on the next day he could not walk without assistance. When he was brought to Kitano Hospital the same day, examination revealed mild right hemiparesis with spared cranial nerve functions. Sensory deficit was minimal and limited to a mild vibratory impairment in both legs. The deep tendon reflexes were generally brisk and the plantar response was extensor on the right. The progression of the weakness was rapid and the neurological findings 2 days later were consistent with transverse myelopathy, with the sensory disturbance at and below the third cervical cord segment (C3). Myelography demonstrated swelling of the mid-cervical spinal cord. CT suggested a somewhat hyperdense lesion in the centre of the swollen cord. The initial examination of the cerebrospinal fluid (CSF) taken on the third day following admission was unremarkable. However, the second CSF test 3 weeks later revealed xanthochromia and a protein level of $4 \cdot 12 \mathrm{~g} / \mathrm{l}$, without pleocytosis.

Following the myelogram the patient developed dyspnoea and respiratory insufficiency and underwent endotracheal intubation. Although he subsequently required a tracheostomy and artificial ventilation, there was no appreciable bulbar palsy. He could change the pattern of ventilation for himself and could stop breathing or even sigh to some degree. Throughout the total of 25 months that the patient remained in hospital there occurred no significant neurological changes, except that the respirator could be withdrawn after 8 months. He eventually died of bronchopneumonia. At necropsy the only significant general abnormalities were the findings in the lungs and nephrosclerosis.

\section{Neuropathology}

The spinal cord at the C5-6 level was discoloured brown and very thin ventro-dorsally. On sectioning, multiloculated old haematomas, surrounded by partly necrotic and sclerotic 


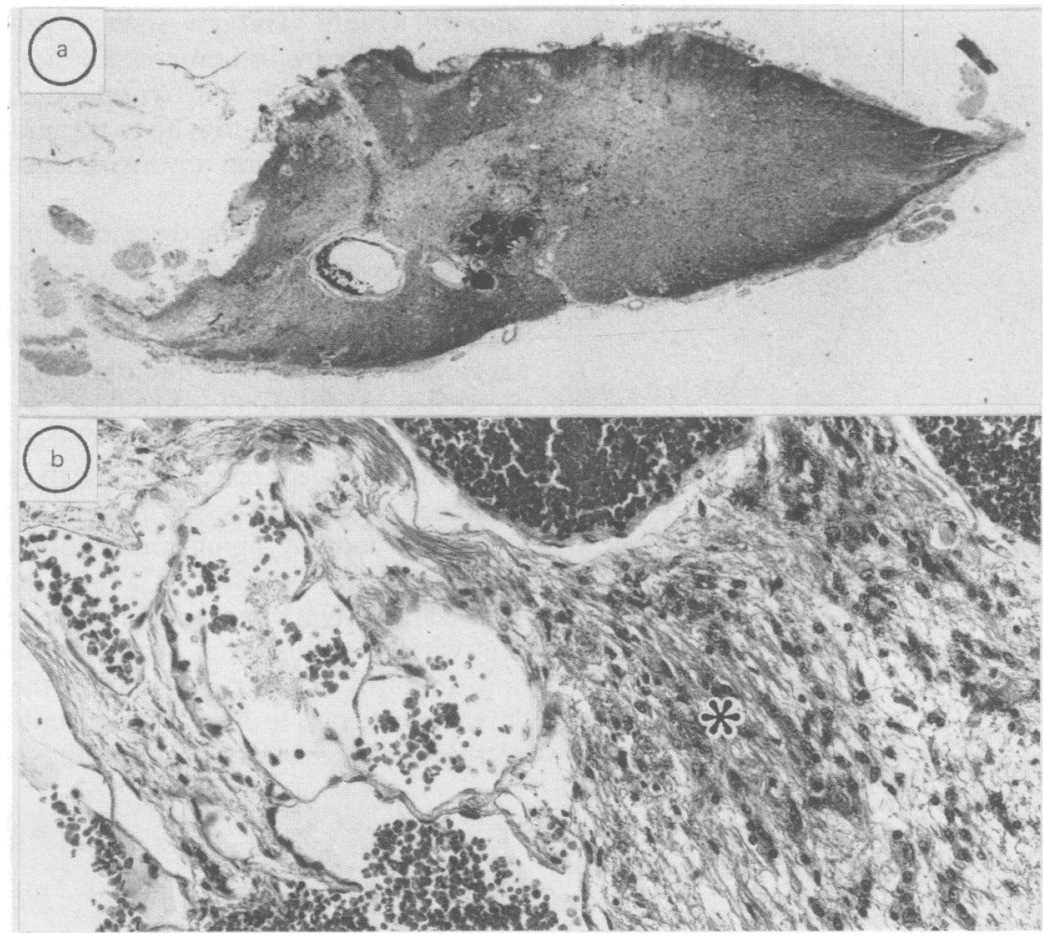

Fig 1 (A) Old haematoma near the central area of cord at $C 5$ which has undergone significant atrophic changes. (B) Higher magnification showing cavernous, thin-walled blood vessels. Numerous haemosiderin-laden macrophages are present (asterisk). $(H \& E,(A) \times 6,(B) \times 170$.)

yellowish lesions, were found near the centre of the spinal cord ftom C5 to C7. No abnormal vascular malformation was found on the surface of the spinal cord or at any level of the cord other than cervical.

Histologically, old haematomas were confirmed around which were grouped about a dozen abnormally-dilated, thinwalled blood vessels, with (and in some cases without) gliotic neural tissue between them, consistent with a ruptured cavernous angioma (fig 1). ${ }^{9}$ The distinction between gray and white matter was obscured and more than half of the crosssectional area of the cord (mainly the right central and posterior regions) was necrotic with fat- or haemosiderinladen macrophages being present. The periphery of the atrophic cord was severely gliotic and the leptomeninges were fibrotic. Axonal swellings were scattered near the lesion. A slit-like region of necrosis, which was presumed to be directly related to the original haemorrhage, could be traced, gradually becoming smaller in size, through the right side of the posterior column up to the dorsal aspect of the caudal medulla.

Ordinary Wallerian (anterograde) tract degeneration was observed rostrally in the posterior columns, posterior and anterior spinocerebellar tracts and spinothalamic tracts, and caudally in the lateral as well as anterior corticospinal tracts. Unexpectedly, the corticospinal tracts showed degeneration in their rostral portions, particularly evident up to the pontomedullary junction (fig 2). Using H\&E, Sudan III, Luxol fast blue-periodic acid Schiff, and modified Bielschowsky stains, ${ }^{10}$ this change could be followed up to the mid-pontine level bilaterally as determined by the presence of fat-laden macrophages, loss of myelin and axons, and gliosis.

The cerebrum and cerebellum were unremarkable both macroscopically and microscopically, except that no typical large pyramidal cells of Betz could be detected in the fifth

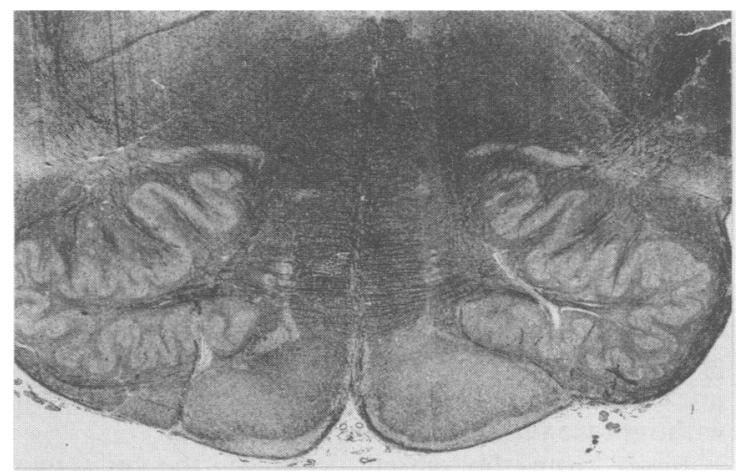

Fig 2 Retrograde pyramidal tract degeneration manifested by myelin pallor in medullary pyramids. (Luxol fast blueperiodic acid Schiff, $\times 6.5$.) 


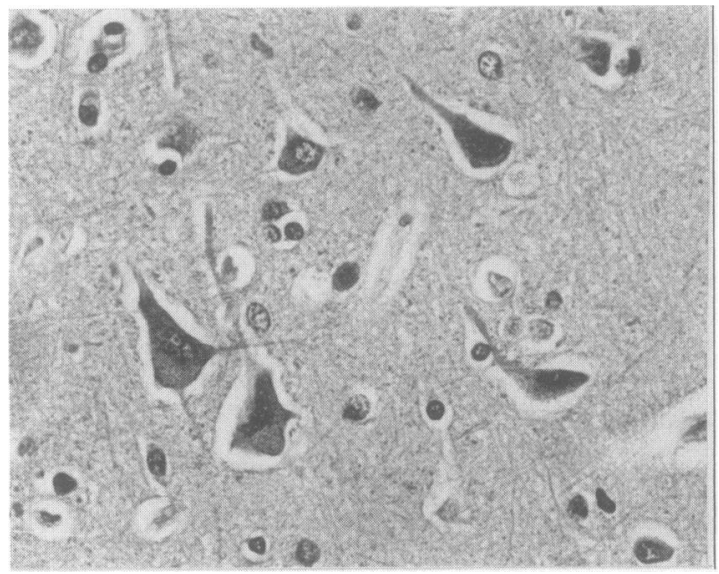

Fig 3 The fifth layer of the motor cortex showing pyramidal cells of modest size. No typical Betz cells, central chromatolysis, or gliosis are seen. (H\&E, × 350.)

layer of the precentral gyrus (fig 3). Neither dying cells nor glial reaction was observed there.

These findings were consistent with retrograde tract degeneration not only because no abnormalities were seen in the upper pontine base, midbrain, internal capsules and precentral motor cortex, but also because of the decreasing severity of the change in the upward direction. It was not possible, however, to detect any histological difference between the anterograde and retrograde corticospinal tract degeneration, except that the severity in the former remained marked as far as the lower lumbar level and could be demonstrated even in the sacral level.

Furthermore, Sudan III clearly demonstrated degradation products, from the caudal pons through to the upper pons, in the central portion of the medial lemniscus, where the location of a so-called aberrant pyramidal pathway has been described (fig 4). ${ }^{5}$ There was no apparent lesion in the medullary medial lemniscus, from which the ascending sensory fibres should reach the pontine level.

\section{Discussion}

Until $1978^{3}$ there had been allegedly only eight human cases of retrograde pyramidal tract degeneration, with Hunt's the earliest in $1904 .{ }^{4}$ In a 24 year old woman with an 18 year history of traumatic lumbar myelopathy, Bronson et al described such degeneration which was traced up to the fifth cervical cord level, along with ordinary Wallerian degeneration in the gracilis, spinocerebellar, and spinothalamic tracts above the lesion. The lumbosacral cord was flattened without recognisable architecture. ${ }^{3}$

Apart from the present instance, the only two previous human cases with retrograde pyramidal tract degeneration reaching up the brainstem have been those of Gombault and Phillipe (cited in reference 4) and of Hunt. ${ }^{4}$ Though such rostral extension is undoubtedly rare in humans, a lesion made in hamsters just rostral to the pyramidal decussation was followed by degeneration up to the midbrain. "In cats, a mid-medullary lesion produced degeneration as high as the pons. ${ }^{12}$

While retrograde tract degeneration has been noted to decrease in severity as it reaches the cell bodies of the origin of the tract, ${ }^{3}$ its histological characteristics remain to be elucidated. Slightly swollen axons and large vacuoles have been described, but are not always present. ${ }^{3}$ In hamsters, shrunken axons and small axonal beads have been noted. " One common finding, however, has been that retrograde degeneration is less compactly sclerotic than Wallerian degeneration. We could not find any significant histological differences, apart from the severity of axonal loss and gliosis, between the Wallerian and retrograde degeneration. Yet we did confirm that Wallerian degeneration did not diminish in severity as far as the lower lumbar level, in contrast to the rather abrupt diminutions of retrograde degeneration above the pontomedullary

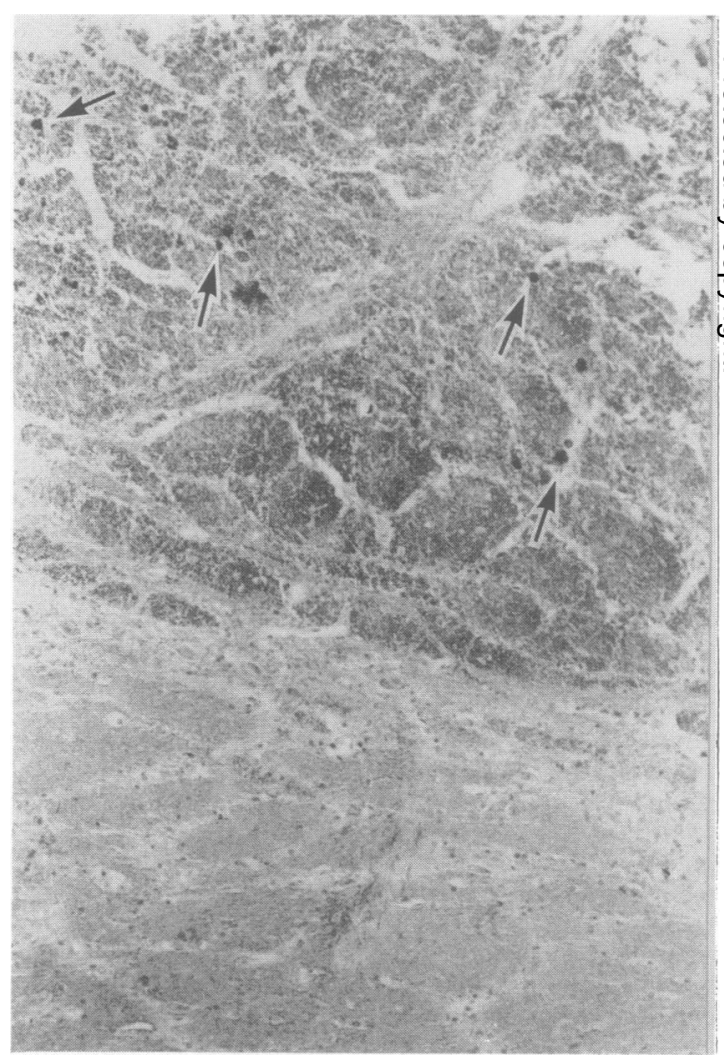

Fig 4 Sudanophilic degradation materials (arrows) in the aberrant pyramidal tract in the central portion of pontine medial lemniscus. (Sudan III, × 90.) 
junction. Similarly to the findings of other workers, ${ }^{2}$ there were no obvious retrograde changes in the posterior columns above, and spinocerebellar tracts below, the lesion.

The restricted nature of retrograde degeneration is compatible with the hypothesis ${ }^{211}$ that the transected pyramidal tract axons undergo retrograde dissolution only to the level of the next most proximal, undamaged axon collateral. Compared with the previous studies, ${ }^{2-4}$ the higher level of retrograde change in this case might be explained if the direct rostral extension of the haematomyelia, up to the caudal medulla, was more significant than it appeared to be at necropsy. Although the mechanisms leading to retrograde degeneration are by no means clear from the reported cases, nor from ours, it might be fruitful to perform detailed studies on a large number of patients dying with chronic spinal cord pathology, such as the study of Fishman. ${ }^{2}$

Another interesting aspect of retrograde change is the fate of the Betz cells. Retrograde neuronal change or axonal reaction, which is frequently alternatively referred to as chromatolysis, ${ }^{1}$ is a well known phenomenon occurring after the severance of the axon. While chromatolysis is typically seen in the spinal anterior horn cells, a different type of change occurs when the neurons and their processes are entirely within the central nervous system..$^{13}$ In the latter case the axonal reaction is characterised by progressive somal atrophy ${ }^{13}$ or neuronal death. ${ }^{14}$ Despite searching through many sections, Betz cells were not found in the precentral gyrus in the present case, and only neurons of modest size were seen in the fifth cortical layer. This appears to imply, as Barron and Dentinger have contended, ${ }^{15}$ that these large cells have undergone atrophic changes without actually dying, since the proximal axons rostral to the pons remained normal in appearance.

Of related interest is the change in the neurons of Clarke's column following rostral cord resection. In a recent report the large neurons were shown to disappear, ${ }^{16}$ but we were unable to confirm such a definite change, though morphometric studies were not carried out. There should be significant metabolic differences underlying these varied types of behaviours between the pyramidal and spinocerebellar tracts. Further studies are needed to confirm or disprove this notion.

The methods employed for the demonstration of degeneration could be important factors in the different observations reported between authors. ${ }^{3}$ Most previous investigators have apparently examined the materials for the presence of myelin pallor. ${ }^{3}$ We found that Sudan III stain was the most reliable and the most useful for the demonstration of faint changes, which were difficult, although not impossible, to find with the other stains we used following careful inspection. Marchi's method, which is favoured by many investigators, ${ }^{17}$ might have been even more sensitive, although its capricious nature is well known.

Finally, the aberrant pyramidal tract is known to be located in the medial lemniscus and is supposed to innervate the cranial nerve nuclei from the third through to the twelfth. ${ }^{5}$ Our case suggests that the aberrant pyramidal tract in the medial lemniscus may contain corticospinal components in a similar region as the corticobulbar fibres and undergo retrograde degeneration along with the ordinary pyramidal tract. Another explanation would be that the corticobulbar fibres were damaged in some way by the direct extension of the haematomyelia up to the caudal medulla. However, the slit-like lesion at this level was so small that the latter possibility cannot be seriously considered. With regard to the absence of degeneration in the medullary medial lemniscus in the present case, it appears reasonable to postulate that at least the major aberrant pyramidal fibres do not pass through there. The significance of this aberrant tract would also be of clinical interest with the advent of highquality MRI.

\section{References}

1 Brodal A. Neurological Anatomy in Relation to Clinical Medicine. 3rd ed. New York: Oxford University Press, 1981:3-45.

2 Fishman PS. Retrograde changes in the corticospinal tract of post-traumatic paraplegics. Arch Neurol 1987;44:1082-4.

3 Bronson R, Gilles FH, Hall J, Hedley-Whyte ET. Long term post-traumatic retrograde corticospinal degeneration in man. Hum Pathol 1978;9:602-7.

4 Hunt JR. The retrograde atrophy of the pyramidal tracts. J Nerv Ment Dis 1904;31:504-12.

5 Crosby EC, Humphrey T, Lauer EW. Correlative anatomy of the nervous system. New York: Macmillan, 1962:262-4.

6 Puvanendran K, Wong PK, Ransome GA. Syndrome of Dejerine's fourth reich. Acta Neurol Scand 1978;57:349-53.

7 Rothstein TL, Alvord EC Jr. Posterior internuclear ophthalmoplegia. A clinicopathological study. Arch Neurol 1971;24:191-202.

8 Shibasaki H, Shida K, Kuroiwa Y. Lateral gaze palsy resulting from brainstem lesion-its classification and relations with internuclear ophthalmoplegia. Clin Neurol (Tokyo) 1974;14:580-7.

9 Jellinger K. Pathology of spinal vascular malformations and vascular tumors. In: Pia HW, Djindjian R, eds. Spinal Angiomas. Advances in Diagnosis and Therapy. Berlin: Springer-Verlag, 1978:18-44.

10 Yamamoto T, Hirano A. A comparative study of modified Bielschowsky, Bodian and thioflavin S stains on Alzheimer's neurofibrillary tangles. Neuropathol 
Appl Neurobiol 1986;12:3-9.

$11 \mathrm{Kalil} \mathrm{K}$, Schneider GE. Retrograde cortical and axonal changes following lesions of the pyramidal tract. Brain Res 1975;89:15-27.

12 Lance JW. Behaviour of pyramidal axons following section. Brain 1954;77:314-24.

13 Barron KD, Dentinger MP, Popp AJ, Mankes R. Neurons of layer $\mathrm{Vb}$ of rat sensorimotor cortex atrophy but do not die after thoracic cord transection. $J$ Neuropathol Exp Neurol 1988;47:62-74.

14 Feringa ER. Gilbertie WJ, Vahlsing HL. Histologic evidence for death of cortical neurons after spinal cord transection. Neurology 1984;34:1002-6.
15 Barron KD, Dentinger MP. Cytologic observations on axotomized feline Betz cells. 1. Qualitative electron microscopic findings. J Neuropathol Exp Neurol 1979;38:128-51.

16 Feringa ER, Pruitt JN II, McBride RL, Vahlsing HL. Changes in number and size of Clarke's column neurons after cord transection. J Neuropathol Exp Neurol 1987;46:695-702.

17 Ikuta F, Makifuchi T, Ohama E, Takeda S, Oyanagi K, Nakashima S, Motegi T. Tract degeneration of the human spinal cord: some observations on ALS and hemispherectomized humans. Adv Neurol Sci (Tokyo) 1982;26:710-36. 